\title{
Schoenberg coefficients and curvature at the origin of continuous isotropic positive definite kernels on spheres
}

\author{
Ahmed Arafat ${ }^{\mathrm{a}}$, Pablo Gregori ${ }^{\mathrm{b}, *}$, Emilio Porcu ${ }^{\mathrm{c}}$ \\ ${ }^{a}$ Department of Mathematics, Mansoura University, Mansoura, Egypt \\ ${ }^{b}$ Instituto Universitario de Matemáticas y Aplicaciones de Castellón, Departamento de \\ Matemáticas, Universitat Jaume I de Castellón, Campus Riu Sec, E-12071, Castellón, \\ Spain \\ ${ }^{c}$ Chair of Spatial Analytics Methods, School of Mathematics, Statistics and Physics, \\ Newcastle University, UK
}

\begin{abstract}
We consider the class $\Psi_{d}$ of continuous functions that define isotropic covariance functions in the $d$-dimensional sphere $\mathbb{S}^{d}$. We provide a new recurrence formula for the solution of Problem 1 in Gneiting (2013b), solved by Fiedler (2013). In addition, we have improved the current bounds for the curvature at the origin of locally supported covariances (Problem 3 in Gneiting (2013b)), which is of applied interest at least for $d=2$.
\end{abstract}

Keywords: Positive definite kernel, Schoenberg coefficients, Gegenbauer polynomials, Isotropic covariance function

2010 MSC: 60G69, 45H05, 33C45

\section{Introduction}

There has been a fervent research activity around positive definite functions on spheres in the last five years Barbosa and Menegatto (2015); Beatson and zu Castell (2017); Castro et al. (2012); Estrade et al. (2019); Fiedler (2013);

${ }_{5}$ Gneiting (2013a); Guella and Menegatto (2016, 2018); Guella et al. (2016a) b. 2018); Massa et al. (2017); Menegatto (2014); Porcu et al. (2016); Trubner and Ziegel (2017); Xu (2018); Ziegel (2014). In Gneiting (2013a), T. Gneiting

\footnotetext{
${ }^{*}$ Corresponding author

Email address: gregori@uji.es (Pablo Gregori)
}

Preprint submitted to Statistics \& Probability Letters 
offers an impressive overview of the problem as well as a number of connections between mathematical, complex and harmonic analysis tools, approximation theory, and the theory of stochastic processes, Gaussian random fields, and geostatistics.

Schoenberg's theorem (Schoenberg, 1942, Thm. 2), in concert with the orthonormality properties of spherical harmonics, characterises positive definite functions over $d$-dimensional spheres of $\mathbb{R}^{d+1}$ that depend on the geodesic (great circle) distance. Such an assumption is termed geodesic isotropy by Porcu et al. (2018), and it is the building block for more sophisticated constructions, such as in Berg and Porcu (2017); Estrade et al. (2019) and Porcu et al. (2016). More technical approaches based on complex spheres and locally compact groups have been proposed in Berg et al. (2017).

Gneiting (2013b) provides a collection of open problems that have inspired mathematicians and statisticians, as it can be seen, for instance, from Fiedler (2013); Berg and Porcu (2017); Massa et al. (2017); Ziegel (2014) as well as from the tour de force in Beatson et al. (2013).

This paper faces two important problems, the former being related to the representation of $d$-Schoenberg's coefficients (see Section 2 below) in terms of 1-Schoenberg coefficients. Such a problem is parenthetical to celebrated Matheron's turning bands operator Matheron (1963) proposed in Euclidean spaces only. In particular, a representation of $d$-Schoenberg coefficients in terms of 1Schoenberg's coefficients was provided by Fiedler (2013) when $d$ is odd, and in terms of 2-Schoenberg's coefficients when $d$ is even. The case of even dimension $d$ and a representation in terms of 1-Schoenberg's coefficients is still elusive, and constitutes one of the challenges and achievements of the present paper.

The latter problem finds instead motivation in atmospheric data assimilation where, quoting Gneiting (2013b), "locally supported isotropic correlation

35 functions are used for the distance-dependent reduction of global scale covariance estimates in ensemble Kalman filter settings Buehner and Charron (2007); Hamill et al. (2001)." Our contribution, in this regard, provides sharper bounds related to the minimum curvature at the origin of compactly supported positive 
definite functions.

The plan of the paper is the following. Section 2 provides the necessary concepts, notation and theoretical tools. Section 3 introduces the statements of Problems 1 and 3 of Gneiting (2013b) and follows with our improvements to their current solutions.

\section{The class $\Psi_{d}$ and $d$-Schoenberg coefficients}

${ }_{45} \quad$ Let $d$ be a positive integer. We consider the $d$-dimensional sphere $\mathbb{S}^{d}$ with unit radius, embedded in $\mathbb{R}^{d+1}$ so that $\mathbb{S}^{d}=\left\{x \in \mathbb{R}^{d+1}:\|x\|=1\right\}$. We define the geodesic or great circle distance as the mapping $\theta: \mathbb{S}^{d} \times \mathbb{S}^{d} \rightarrow[0, \pi]$ defined through $\theta(\xi, \eta)=\arccos (\langle\xi, \eta\rangle)$, with $\langle\cdot, \cdot\rangle$ denoting the classical dot product. Throughout, we use the abuse of notation $\theta$ for $\theta(\xi, \eta)$ whenever there is no confusion. We also consider the Hilbert sphere $\mathbb{S}^{\infty}=\left\{x \in \mathbb{R}^{\mathbb{N}}\right.$ : $\|x\|=1\}$. We say that the function $C: \mathbb{S}^{d} \times \mathbb{S}^{d} \rightarrow \mathbb{R}$ is positive definite if $\sum_{i, j=1}^{n} \alpha_{i} \alpha_{j} C\left(\mathbf{x}_{i}, \mathbf{x}_{j}\right) \geq 0$, for any $\alpha_{1}, \ldots, \alpha_{n} \in \mathbb{R}$ and for every $\mathbf{x}_{1}, \ldots, \mathbf{x}_{n} \in \mathbb{S}^{d}$.

We denote by $C_{n}^{\lambda}$ the $n$-th Gegenbauer polynomial of order $\lambda>0$, uniquely identified through the intrinsic relation

$$
\frac{1}{\left(1+r^{2}-2 r \cos \theta\right)^{\lambda}}=\sum_{n=0}^{\infty} r^{n} C_{n}^{\lambda}(\cos \theta), \quad \theta \in[0, \pi],
$$

where $r \in(-1,1)\left(\right.$ DLMF Eq. 18.12.4). The first three polynomials are $C_{0}^{\lambda}(x)=$ $1, C_{1}^{\lambda}(x)=2 \lambda x$ and $C_{2}^{\lambda}(x)=2 \lambda(\lambda+1) x^{2}-\lambda$, for $x \in[-1,1]$ (DLMF, Eq. 19.8.1). It is of fundamental importance that (DLMF, Eq. 18.14.4)

$$
\left|C_{n}^{\lambda}(x)\right| \leq \frac{\Gamma(n+2 \lambda)}{n ! \Gamma(2 \lambda)}=C_{n}^{\lambda}(1), \quad x \in[-1,1] .
$$

The trigonometric expansion in the following lemma is crucial for the solution of our first problem. We recall the notation of the rising factorial $(x)_{m}:=$ $x(x+1) \cdots(x+m-1)$ for any real number $x$ and any non negative integer length $m$, with the convention $(x)_{0}=1$. 
Lemma 1. Let $n \geq 1$ be an integer, $\lambda>0$ and $0<\theta<\pi$. Then the expansion $(\sin \theta)^{2 \lambda-1} C_{n}^{\lambda}(\cos \theta)=\frac{2^{2-2 \lambda} \Gamma(n+2 \lambda)}{\Gamma(\lambda) \Gamma(n+\lambda+1)} \sum_{\mu=0}^{\infty} \frac{(1-\lambda)_{\mu}(n+1)_{\mu}}{\mu !(n+\lambda+1)_{\mu}} \sin (n+2 \mu+1) \theta$

holds, and reduces to a finite sum (up to $\mu=\lambda-1$ ) whenever $\lambda$ is an integer.

This result is given in (Szegö, 1939, p. 93, Eq. 4.9.22) and proved for $\lambda>0$, $\lambda \neq 1,2,3, \ldots$ and $0<\theta<\pi$. The remaining case can be proved by induction on $\lambda \in\{1,2, \ldots\}$.

Let $\Psi_{d}$ be the class of continuous mappings $\psi:[0, \pi] \rightarrow \mathbb{R}$ with $\psi(0)=1$ such that the continuous functions $C: \mathbb{S}^{d} \times \mathbb{S}^{d} \rightarrow \mathbb{R}$ defined through $C(\xi, \eta)=$ $\psi(\theta(\xi, \eta))$ are positive definite. The dimension $d$ and the parameter $\lambda$ in Eq. 1 are related by $\lambda:=(d-1) / 2$, and in the sequel, for ease of notation, we use one or the other interchangeably. Schoenberg (1942) characterised the positive definite functions defined on the spheres of any dimension.

Theorem 2.1. Schoenberg (1942) A necessary and sufficient condition for a continuous mapping $\psi:[0, \pi] \rightarrow \mathbb{R}$, with $\psi(0)=1$ to belong to the class $\Psi_{d}$ is that the ultraspherical expansion

$$
\sum_{n=0}^{\infty}\left\{\frac{(n+\lambda) \Gamma(\lambda)}{\Gamma\left(\lambda+\frac{1}{2}\right) \Gamma\left(\frac{1}{2}\right)} \frac{\Gamma(n+1) \Gamma(2 \lambda)}{\Gamma(n+2 \lambda)} \cdot \int_{0}^{\pi} C_{n}^{\lambda}\left(\cos \theta^{\prime}\right) \psi\left(\theta^{\prime}\right) \sin ^{2 \lambda} \theta^{\prime} \mathrm{d} \theta^{\prime}\right\} C_{n}^{\lambda}(\cos \theta)
$$

has non-negative coefficients and converges absolutely and uniformly to $\psi(\theta)$ throughout $0 \leq \theta \leq \pi$.

Gneiting (2013a) used Theorem 2.1 to characterise the members of class $\Psi_{d}$ through the representation

$$
\psi(\theta)=\sum_{n=0}^{\infty} b_{n, d} \frac{C_{n}^{\lambda}(\cos \theta)}{C_{n}^{\lambda}(1)}, \quad \theta \in[0, \pi],
$$

with $\left\{b_{n, d}\right\}_{n=0}^{\infty}$ being a uniquely identified probability mass system. We follow Daley and Porcu (2014) and Ziegel (2014) when referring to $b_{n, d}$ as $d$-Schoenberg coefficients. 
The classes $\Psi_{d}$ are nested, with the inclusion relation $\Psi_{1} \supset \Psi_{2} \supset \cdots \supset$ $\Psi_{\infty}:=\bigcap_{d \geq 1} \Psi_{d}$ being strict, and where $\Psi_{\infty}$ has a direct relation to the Hilbert sphere as previously defined.

Gneiting (2013a) and Beatson et al. (2013) obtain recurrence formulae that allow to write any coefficient $b_{n, d}$ as a linear combination of $b_{n, d-2}$ and $b_{n+2, d-2}$. By applying recursivity, each coefficient $b_{n, d}$ can be finally written, when $d$ is

80 odd, as a linear combination of 1-Schoenberg coefficients $\left\{b_{n+2 k, 1}\right\}_{k=0}^{\lfloor d / 2\rfloor}$, and when $d$ is even, as a linear combination of 2-Schoenberg coefficients $\left\{b_{n+2 k, 2}\right\}_{k=0}^{d / 2}$.

Using the orthogonality of Gegenbauer polynomials, we can identify coefficients of Eq. (3) and (4) and get (Gneiting, 2013a, Cor. 2)

$$
b_{n, d}=\frac{(n+\lambda) \Gamma(\lambda)}{\Gamma(\lambda+1 / 2) \Gamma(1 / 2)} \int_{0}^{\pi} C_{n}^{\lambda}(\cos \theta) \psi(\theta)(\sin \theta)^{2 \lambda} \mathrm{d} \theta .
$$

where as usual $\lambda:=(d-1) / 2$.

We recall that the 1-Schoenberg coefficients are the Fourier coefficients for even functions:

$$
b_{0,1}:=\frac{1}{\pi} \int_{0}^{\pi} \psi(\theta) \mathrm{d} \theta, \quad b_{n, 1}:=\frac{2}{\pi} \int_{0}^{\pi} \psi(\theta) \cos (n \theta) \mathrm{d} \theta, \quad(n \geq 1) .
$$

\section{Gneiting's problems and current solutions}

\subsection{Statements of the problems}

We now expose the problems faced in the paper together with their partial solutions.

Problem 1. Gneiting, 2013b, Problem 1) Let $n \geq 0$ and $k \geq 1$ be integers. Find the coefficients $a_{n, 1}, \ldots, a_{n, k}$ in the expansion $b_{n, 2 k+1}=\sum_{i=0}^{k} a_{n, i} b_{n+2 i, 1}$ associated to the $(2 k+1)$-Schoenberg coefficients in terms of Fourier coefficients $b_{n, 1}, \ldots, b_{n+2 k, 1}$. Similarly, find the $(2 k+2)$-Schoenberg coefficients in terms of the 2 -Schoenberg coefficients $b_{n, 2}, b_{n+2,2}, \ldots, b_{n+2 k, 2}$.

In order to state Problem 2, we follow Gneiting (2013a) when calling $\Psi_{d}^{c}$ the subclass of $\Psi_{d}$ having members $\psi$ that vanish for any $\theta \geq c$, with $c \in(0, \pi]$. When $c<\pi$, then any member of $\Psi_{d}^{c}$ is called locally supported, otherwise it is called globally supported. 
Problem 2. Gneiting, 2013b, Problem 3) For an integer $d \geq 1$, and for a given $c \in(0, \pi]$, find

$$
a_{d}^{c}:=\inf _{\psi \in \Psi_{d}^{c}}\left(-\psi^{\prime \prime}(0)\right) .
$$

The application on atmospheric data motivates the search of a member of the class $\Psi_{2}^{c}$ with minimal curvature at the origin.

Some comments are in order. The solution of Problem 1 requires the use of recursive formulae for the Gegenbauer polynomials and a constructive argument that will be exposed subsequently. For Problem 2, we assume the existence of $\psi^{\prime \prime}(0)$ (considered in the one-sided sense), which is equivalent to the convergence of $\sum_{n} n^{2} b_{n, d}$, as shown in (Trubner and Ziegel, 2017, Lemm. 2.1). Our approach relies on considering $\widetilde{\Psi}_{d}^{c}$, the subclass of $\Psi_{d}$ given by those members $\psi \in \Psi_{d}$ such that $\psi(c)=0$.

Another relevant comment is that Theorems 2 and 3 in Gneiting (2013a) provide the upper bound $a_{d}^{c} \leq \frac{1}{c^{2}} \frac{4}{d} j_{\frac{d-2}{2}}^{2}$, where $j_{\nu}$ denotes the first positive zero of the Bessel function $J_{\nu}$.

According to Ehm et al. (2004) the constant $a_{d}^{c}$ in Euclidean spaces depend on Boas-Kac roots, but Ziegel (2014) claims that the construction of these roots for positive definite functions on spheres remains an open problem. This makes the problem mathematically more interesting, and certainly tricky.

\subsection{Main results}

The next proposition gives a general expression of $d$-Schoenberg coefficients $b_{n, d}$ as a linear combination of the Fourier (cosine) coefficients for arbitrary dimension $d$. It is necessary to mention that Problem 2 of Gneiting (2013b) was completely solved for the first time by J. Fiedler in (Fiedler. 2013, Thms. 2.1\&2.4), using induction on the dimension $d$. He determined the weights of the combination of the Fourier cosine coefficients for odd $d$, and found the weights of the combination of the Legendre coefficients for even $d$. Our result only covers the odd dimension case, and gives an equivalent expression. However, it contributes a combination as an infinite series of Fourier cosine coefficients for the even dimension case. 
Proposition 1. Let $d>1$ be an integer, and let $\lambda=(d-1) / 2$. Then,

$$
\begin{aligned}
b_{n, d}=\frac{\sqrt{\pi} \Gamma(n+2 \lambda)}{2^{2 \lambda} \Gamma(\lambda+1 / 2) \Gamma(n+\lambda)}\left[b_{n, 1}^{*}\right. & \\
& \left.-\lambda \sum_{\mu=1}^{\infty} \frac{(1-\lambda)_{\mu-1}(n+1)_{\mu-1}(n+2 \mu)}{\mu !(n+\lambda+1)_{\mu}} b_{n+2 \mu, 1}\right],
\end{aligned}
$$

for $n \geq 0$, where $b_{n, 1}^{*}=b_{n, 1}$ when $n \geq 1$ and $b_{0,1}^{*}=2 b_{0,1}$. If $d$ is odd, the expression involves only a finite number of coefficients, i.e., $b_{n, 1}^{*}, b_{n+2,1}, \ldots, b_{n+2 \lambda, 1}$.

Proof. By plugging Eq. (2) into Eq. (5), taking into account Eq. (1), and using again the product-to-sum trigonometric identities, we get

$$
\begin{aligned}
b_{n, d} & =\alpha_{\lambda, n} \int_{0}^{\pi}\left[\sum_{\mu=0}^{\infty} \beta_{\lambda, n, \mu} \sin (n+2 \mu+1) \theta \sin \theta\right] \psi(\theta) \mathrm{d} \theta \\
& =\frac{\alpha_{\lambda, n}}{2} \int_{0}^{\pi}\left[\beta_{\lambda, n, 0} \cos (n \theta)+\sum_{\mu=1}^{\infty}\left[\beta_{\lambda, n, \mu}-\beta_{\lambda, n, \mu-1}\right] \cos (n+2 \mu) \theta\right] \psi(\theta) \mathrm{d} \theta,
\end{aligned}
$$

where

$$
\alpha_{\lambda, n}:=\frac{2^{2-2 \lambda} \Gamma(n+2 \lambda)}{\Gamma(\lambda+1 / 2) \Gamma(1 / 2) \Gamma(n+\lambda)} \quad \text { and } \quad \beta_{\lambda, n, \mu}:=\frac{(1-\lambda)_{\mu}(n+1)_{\mu}}{\mu !(n+\lambda+1)_{\mu}} .
$$

If $\lambda$ is an integer (i.e. $d$ is odd), the series is a finite sum (up to index $\mu=\lambda-1)$. Otherwise, we need to verify the uniform convergence of the series in $(0, \pi)$, in order to exchange the integral and the series signs in (9).

On the one hand, it is easy to check that $\beta_{\lambda, n, 0}=1$, and

$$
\beta_{\lambda, n, \mu}-\beta_{\lambda, n, \mu-1}=\frac{-\lambda(n+2 \mu)}{\mu(n+\lambda+\mu)} \beta_{\lambda, n, \mu-1},
$$

for all $n$ and $\lambda$, and $\mu=1,2,3, \ldots$ On the other hand, if $\lambda$ is not an integer, and $\mu$ is larger than $n$,

$$
\beta_{\lambda, n, \mu-1}=\frac{(1-\lambda)(2-\lambda) \cdots(n-\lambda)}{1 \cdot 2 \cdots n} \cdot \frac{\mu(\mu+1) \cdots(\mu+n-1)}{(\mu-\lambda)(\mu-\lambda+1) \cdots(\mu+n+\lambda-1)}
$$

(just expand the rising factorials in the definition of $\beta_{\lambda, n, \mu-1}$ and cancel factors 
appropriately). Now,

$$
\begin{aligned}
\left|\sum_{\mu=1}^{\infty}\left[\beta_{\lambda, n, \mu}-\beta_{\lambda, n, \mu-1}\right] \cos (n+2 \mu) \theta\right| & \leq \sum_{\mu=1}^{\infty}\left|\beta_{\lambda, n, \mu}-\beta_{\lambda, n, \mu-1}\right| \\
& =\sum_{\mu=1}^{\infty} \frac{\lambda(n+2 \mu)}{\mu(n+\lambda+\mu)} \beta_{\lambda, n, \mu-1}
\end{aligned}
$$

where the last series involves a quotient of polynomials in $\mu$ (for each fixed $\lambda$ and $n$ ) of respective degrees $n+1$ and $n+2 \lambda+2(=n+d+1)$. Hence the uniform convergence of the function series.

After exchanging series and integral in (9), we use the definitions of 1Schoenberg coefficients (6): for $n>0$ we get

$$
b_{n, d}=\frac{\pi \alpha_{\lambda, n}}{4}\left[b_{n, 1}+\sum_{\mu=1}^{\infty}\left[\beta_{\lambda, n, \mu}-\beta_{\lambda, n, \mu-1}\right] b_{n+2 \mu, 1}\right]
$$

while for the special case $n=0$ we have

$$
b_{0, d}=\frac{\pi \alpha_{\lambda, 0}}{4}\left[2 b_{0,1}+\sum_{\mu=1}^{\infty}\left[\beta_{\lambda, 0, \mu}-\beta_{\lambda, 0, \mu-1}\right] b_{2 \mu, 1}\right],
$$

Finally, plugging 10 into these expressions, and making use of notation $b_{n, 1}^{*}=$ $b_{n, 1}$ when $n \geq 1$ and $b_{0,1}^{*}=2 b_{0,1}$, we get the final result.

We are now able to face Problem 2, where a formal statement for a partial solution is exposed in the following.

Proposition 2. Let $d>1$ be an integer. Then:

(i) $a_{d}^{c} \geq \frac{1}{1-\cos c}$ if $c \in[\pi / 2, \pi]$.

(ii) $a_{d}^{c} \geq \frac{(d+1)(\cos c)(2-\cos c)+1}{(1-\cos c)((d+1) \cos c+1)}$ if $c \in\left[\arccos \sqrt{\frac{1}{d+1}}, \pi / 2\right]$.

Proof. It is easy to check Beatson et al. (2013) that $-\psi^{\prime \prime}(0)=\frac{1}{d} \sum_{n=1}^{\infty} n(n+d-$ 1) $b_{n, d}$ for any $\psi \in \Psi_{d}$ with associated $d$-Schoenberg coefficients $\left\{b_{n, d}\right\}_{n=0}^{\infty}$ and holding $\sum_{n} n^{2} b_{n, d}<\infty$. Since the sequence $\left\{b_{n, d}\right\}_{n=0}^{\infty}$ forms a probability mass system, $-\psi^{\prime \prime}(0)$ shall be smaller for functions $\psi$ whose mass is concentrated in lower index coefficients. The search of these functions shall provide sharper bounds for the infimum $a_{d}^{c}$ in Equation (7). 
The set $\Psi_{d}^{c}$ is difficult to tackle, because locally supported functions have an infinite number of non null $d$-Schoenberg coefficients. In view of this, we consider $\Psi_{d}^{c}$ as a subset of the more amenable set $\widetilde{\Psi}_{d}^{c}:=\left\{\psi \in \Psi_{d}: \psi(c)=0\right\}$, of functions having at least one zero at the fixed value $\theta=c$. Now, let us denote $\widetilde{a}_{d}^{c}:=\inf _{\psi \in \widetilde{\Psi}_{d}^{c}}\left[-\psi^{\prime \prime}(0)\right]$. Obviously, we have $a_{d}^{c} \geq \widetilde{a}_{d}^{c}$ since $\Psi_{d}^{c} \subset \widetilde{\Psi}_{d}^{c}$, and the latter value is attainable at a known function for a range of values of $c$, as we shall show. In order to get $\widetilde{a}_{d}^{c}$ we need to solve the pair of equations

$$
\sum_{n=0}^{\infty} b_{n, d}=1 \quad \text { and } \quad \sum_{n=0}^{\infty} b_{n, d} \frac{C_{n}^{\lambda}(\cos c)}{C_{n}^{\lambda}(1)}=0
$$

subject to the restriction $\left\{b_{n, d}\right\}_{n=0}^{\infty} \subset[0, \infty)$. As already stated, we shall check the values for functions with mass concentrated into the first coefficients. The constant function (i.e., $b_{n, d}=0$ for $n \geq 1$ ) is clearly out of $\widetilde{\Psi}_{d}^{c}$. Thus, we check functions with $b_{n, d}=0$ for $n \geq 2$. Using Eq. 11) we get the single function

$$
\psi_{c}(\theta)=\frac{-\cos c}{1-\cos c}+\frac{1}{1-\cos c} \cos \theta, \quad \theta \in[0, \pi],
$$

and a sufficient condition for $\psi_{c}$ to belong to the class $\widetilde{\Psi}_{d}^{c}$ is that $c \in[\pi / 2, \pi]$, 145 with $-\psi_{c}^{\prime \prime}(0)=\frac{1}{1-\cos c}$. Hence, for $c \in[\pi / 2, \pi]$ we have that $\psi_{c} \in \widetilde{\Psi}_{d}^{c}$, leading to $\widetilde{a}_{d}^{c}=1 /(1-\cos c)$.

For $c \in[0, \pi / 2]$, we have no members of $\widetilde{\Psi}_{d}^{c}$ with $b_{n, d}=0$ for $n \geq 2$, and we shall look for functions with $b_{n, d}=0$ for $n \geq 3$. Using again the system (11), we get the set of functions that can be written as

$$
\begin{aligned}
\psi_{\beta}(\theta)=- & \frac{\cos c}{1-\cos c}+\frac{(d+1) \cos c+1}{d} \beta \\
& +\left(\frac{1}{1-\cos c}-\frac{(d+1)(1+\cos c)}{d} \beta\right) \cos \theta+\beta \frac{(d+1) \cos ^{2} \theta-1}{d},
\end{aligned}
$$

$\theta \in[0, \pi]$, indexed by a parameter $\beta:=b_{2, d}$. The non negativity restriction of their coefficients turns into the inequality

$$
\frac{d \cos c}{(1-\cos c)((d+1) \cos c+1)} \leq \beta \leq \frac{d}{(d+1) \sin ^{2} c},
$$

which leads to a non empty set of values only if $c \geq \arccos \sqrt{\frac{1}{d+1}}$, and $\widetilde{a}_{d}^{c}$ is attained for $\psi_{\beta}$ when $\beta$ attaches to the left-hand side of inequality 12. This completes the proof. 
This strategy might lead to values of $\widetilde{a}_{d}^{c}$ for a wider range of values $c$, by using functions with $b_{n, d}=0$ for $n \geq 4$, and so on, but we have not explored further this line because of the complexity of equations. Another way (yet unexplored) of improving the lower bounds is using slightly more complex auxiliary sets $\Psi_{d}^{\left(c, c^{\prime}\right)}$ of functions having at least two zeros, or even more. We could find no examples of members of this subclass.

Finally, we show formulae for the 2-Schoenberg coefficients of the exponential and Askey families, whose derivation requires simple techniques, but perseverance, and that, up to our knowledge, are not yet published.

Example 1. The 2-Schoenberg coefficients of the exponential family, given by functions $\psi_{\alpha}(\theta)=\exp \left(-\frac{\theta}{\alpha}\right), \theta \in[0, \pi]$, and parameter $\alpha>0$, are given by

$$
\begin{aligned}
& b_{n, 2}= \frac{2 n+1}{2^{1-n}}\left\{\sum _ { m \equiv 0 ( \operatorname { m o d } 2 ) } ^ { n } \left[\left(\begin{array}{c}
n \\
m
\end{array}\right)\left(\begin{array}{c}
\frac{n+m-1}{2} \\
n
\end{array}\right) \frac{\left(1+\mathrm{e}^{-\frac{\pi}{\alpha}}\right)}{(m+1) 2^{m}} .\right.\right. \\
&\left(2^{m}-\sum_{k=0}^{\frac{m}{2}} \frac{1}{(2 k+1)^{2} \alpha^{2}+1}\left(\begin{array}{c}
m+1 \\
\frac{m-2 k}{2}
\end{array}\right)\right)+\sum_{m \equiv 1(\bmod 2)}^{n}\left[\left(\begin{array}{c}
n \\
m
\end{array}\right)\left(\begin{array}{c}
\frac{n+m-1}{2} \\
n
\end{array}\right)\right. \\
&\left.\left.\quad \cdot \frac{\left(1-\mathrm{e}^{-\frac{\pi}{\alpha}}\right)}{(m+1) 2^{m}} \cdot\left(2^{m}-\frac{1}{2}\left(\begin{array}{c}
m+1 \\
\frac{m+1}{2}
\end{array}\right)-\sum_{k=1}^{\frac{m+1}{2}} \frac{1}{4 k^{2} \alpha^{2}+1}\left(\begin{array}{c}
m+1 \\
\frac{m-2 k+1}{2}
\end{array}\right)\right)\right]\right\} .
\end{aligned}
$$

Example 2. The Askey family Askey (1973) is given by functions $\psi_{\alpha, \tau}(\theta)=$ ${ }_{160}\left(1-\frac{\theta}{\alpha}\right)_{+}^{\tau}$ for $\theta \in[0, \pi]$, and parameters $\alpha, \tau>0$. For $\alpha>0$ and $\tau \geq(d+$ 1)/2, fuctions $\psi_{\alpha, \tau}$ belong to the class $\Psi_{d}$ Gneiting (2013a). The 2-Schoenberg coefficients of $\psi_{\alpha, 2}$ are given by:

$$
\begin{aligned}
& b_{n, 2}=(2 n+1) 2^{n-1}\left\{\sum _ { m \equiv 0 ( m o d 2 ) } ^ { n } \left[( \begin{array} { c } 
{ n } \\
{ m }
\end{array} ) ( \begin{array} { c } 
{ \frac { n + m - 1 } { 2 } } \\
{ n }
\end{array} ) \left(\frac{1}{m+1}+\frac{1}{(m+1) \alpha^{2} 2^{m-1}} .\right.\right.\right. \\
& \left.\left.\sum_{k=0}^{\frac{m}{2}}\left(\begin{array}{c}
m+1 \\
k
\end{array}\right) \frac{\cos (m-2 k+1) \alpha-1}{(m-2 k+1)^{2}}\right)\right]+\sum_{m \equiv 1(m o d 2)}^{n}\left(\begin{array}{c}
n \\
m
\end{array}\right)\left(\begin{array}{c}
\frac{n+m-1}{2} \\
n
\end{array}\right)\left[\frac{1}{m+1}\right. \\
& \left.\left.-\frac{1}{(m+1) 2^{m+1}}\left(\begin{array}{c}
m+1 \\
\frac{m+1}{2}
\end{array}\right)+\frac{1}{(m+1) \alpha^{2} 2^{m-1}} \sum_{k=0}^{\frac{m-1}{2}}\left(\begin{array}{c}
m+1 \\
k
\end{array}\right)\left[\frac{\cos (m-2 k+1) \alpha-1}{(m-2 k+1)^{2}}\right]\right]\right\} .
\end{aligned}
$$




\section{Acknowledgements}

We are indebted to Jochen Fiedler for valuable discussions during the prepa-

165

Funding: Ahmed Arafat and Pablo Gregori's research are supported by Spanish Ministerio de Economía, Industria y Competitividad ([project MTM201678917-R]) and Universitat Jaume I de Castellón ([project P1·1B2015-40]). Emilio Porcu is supported by Proyecto Fondecyt [number 1170290].

\section{References}

Askey, R., 1973. Radial Characteristics Functions. Technical Report. DTIC Document.

Barbosa, V., Menegatto, V., 2015. Generalized convolution roots of positive definite kernels on complex spheres. Symmetry Integr Geom 11, 13.

Beatson, R., zu Castell, W., 2017. Dimension hopping and families of strictly positive definite zonal basis functions on spheres. J Approx Theory 221, 2237.

Beatson, R.K., zu Castell, W., Xu, Y., 2013. A Pólya criterion for (strict) positive-definiteness on the sphere. IMA J Num Anal 34, 550-568.

Berg, C., Peron, A., Porcu, E., 2017. Orthogonal expansions related to compact Gelfand pairs. Expo Math .

Berg, C., Porcu, E., 2017. From Schoenberg coefficients to Schoenberg functions. Constr Approx 45, 217-241.

Buehner, M., Charron, M., 2007. Spectral and spatial localization of 185 background-error correlations for data assimilation. Q J Roy Meteor Soc $133,615-630$.

Castro, M., Menegatto, V., Oliveira, C., 2012. Laplace-Beltrami differentiability of positive definite kernels on the sphere. Acta Math Sin Engl Ser 29, 93-104. 
Daley, D., Porcu, E., 2014. Dimension walks and Schoenberg spectral measures. Proc Amer Math Soc 142, 1813-1824.

DLMF, 2019. NIST Digital Library of Mathematical Functions.

n http://dlmf.nist.gov/, Release 1.0.22 of 2019-03-15. URL: http: //dlmf.nist.gov/, f. W. J. Olver, A. B. Olde Daalhuis, D. W. Lozier, B. I. Schneider, R. F. Boisvert, C. W. Clark, B. R. Miller and B. V. Saunders, eds.

Ehm, W., Gneiting, T., Richards, D., 2004. Convolution roots of radial positive definite functions with compact support. Trans Amer Math Soc 356, 46554685 .

Estrade, A., Farias, A., Porcu, E., 2019. Covariance functions on spheres cross time: Beyond spatial isotropy and temporal stationarity. Stat Probabil Lett $151,1-7$.

Fiedler, J., 2013. From Fourier to Gegenbauer: Dimension walks on spheres. arXiv e-prints, arXiv:1303.6856.

Gneiting, T., 2013a. Strictly and non-strictly positive definite functions on spheres. Bernoulli 19, 1327-1349.

Gneiting, T., 2013b. Strictly and non-strictly positive definite functions on

घ spheres: online supplement. Available at https://projecteuclid.org/ download/suppdf_1/euclid.bj/1377612854.

Guella, J., Menegatto, V., 2016. Strictly positive definite kernels on a product of spheres. J Math Anal Appl 435, 286-301.

Guella, J., Menegatto, V., 2018. Unitarily invariant strictly positive definite kernels on spheres. Positivity 22, 91-103.

Guella, J., Menegatto, V., Peron, A., 2016a. An extension of a theorem of Schoenberg to products of spheres. Banach J Math Anal 10, 671-685. 
Guella, J., Menegatto, V., Peron, A., 2016b. Strictly positive definite kernels on a product of spheres II. Symmetry Integr Geom 12, 15.

Guella, J., Menegatto, V., Porcu, E., 2018. Strictly positive definite multivariate covariance functions on spheres. J Multivariate Anal 166, 150-159.

Hamill, T.M., Whitaker, J.S., Snyder, C., 2001. Distance-dependent filtering of background error covariance estimates in an ensemble kalman filter. Mon Weather Rev 129, 2776-2790.

Massa, E., Peron, A., Porcu, E., 2017. Positive definite functions on complex spheres and their walks through dimensions. Symmetry Integr Geom 13, 16.

Matheron, G., 1963. Principles of Geostatistics. Econ Geol 58, 1246-1266.

225 Menegatto, V., 2014. Differentiability of bizonal positive definite kernels on complex spheres. J Math Anal Appl 412, 189-199.

Porcu, E., Alegria, A., Furrer, R., 2018. Modeling temporally evolving and spatially globally dependent data. International Statistical Review 86, 344377.

230 Porcu, E., Bevilacqua, M., Genton, M., 2016. Spatio-temporal covariance and cross-covariance functions of the great circle distance on a sphere. J Am Stat Assoc 111, 888-898.

Schoenberg, I.J., 1942. Positive definite functions on spheres. Duke Math J 9, 96-108.

Szegő, G., 1939. Orthogonal polynomials. American Mathematical Society.

Trubner, M., Ziegel, J., 2017. Derivatives of isotropic positive definite functions on spheres. Proc Am Math Soc 145, 3017-3031.

Xu, Y., 2018. Positive definite functions on the unit sphere and integrals of Jacobi polynomials. Proc Am Math Soc 146, 2039-2048.

${ }_{240}$ Ziegel, J., 2014. Convolution roots and differentiability of isotropic positive definite functions on spheres. Proc Amer Math Soc 142, 2063-2077. 